\title{
Thermodynamics of QCD at large quark chemical potential
}

\section{Andreas Gerhold}

Department of Physics, North Carolina State University, Raleigh, NC 27695, USA

E-mail: agerholeunity.ncsu.edu

\section{Andreas Ipp}

ECT*, Villa Tambosi, I-38050 Villazzano Trento, Italy

E-mail: ipp@ect.it

\section{Anton Rebhan*}

Institut für Theoretische Physik, Technische Universität Wien, A-1040 Vienna, Austria

E-mail: rebhana@hep.itp.tuwien.ac.at

\begin{abstract}
We review the existing weak-coupling results on the thermodynamic potential of deconfined QCD at small and large quark chemical potential and compare with results from lattice gauge theory as well as the exactly solvable case of large- $N_{f}$ QCD. We also discuss the new analytical results on non-Fermi-liquid effects in entropy and specific heat as well as in dispersion laws of quark quasiparticles at large quark chemical potential.
\end{abstract}

29th Johns Hopkins Workshop on Current Problems in Particle Theory: Strong Matter in the Heavens 1-3 August 2005

Budapest

* Speaker. 


\section{Introduction}

Deep in the deconfined phase of QCD, with either temperature $T$ or quark chemical potential $\mu$ much larger than the QCD scale $\Lambda_{\mathrm{QCD}}$, asymptotic freedom eventually leads to a strong coupling constant $g$ that is sufficiently small to permit the use of perturbation theory for calculating the thermodynamics of quarks and gluons under extreme conditions. Indeed, much effort has been invested in calculating the thermodynamic potential of QCD at high temperature. It is by now known up to and including order $g^{6} \log (g)[1,2,3,4]$. These calculations require resummations of ordinary perturbation theory to cope with infrared problems, which is done most elegantly and efficiently by means of effective field theories [5]. Up to and including order $g^{6} \log (g)$ one can in fact set up an expansion into Feynman diagrams, but this breaks down at the order $g^{6}$, which receives nonperturbative contributions from the chromomagnetostatic sector. However, already the expansion up to that point suffers from unusually poor convergence properties and a strong dependence on the renormalization point even at temperatures many orders of magnitude higher than $\Lambda_{\mathrm{QCD}}$, which seems to reduce calculations of this kind to a purely academic enterprise. The perturbative expansion appears to become reliable only for coupling constants so small that the thermodynamic potential is anyway very well approximated by the Stefan-Boltzmann result for a noninteracting plasma.

However, this problem is not specific to QCD at high temperature with its nonperturbative magnetostatic sector. Similarly poor convergence behaviour appears also in simple scalar field theory [6], and even in the case of large- $N \phi^{4}$ theory [7], where all interactions can be resummed in a local thermal mass term. As soon as one starts expanding out in a series of powers and logarithms of the coupling, the result for the thermodynamic potential goes wild, whereas the exact result that is available for the large- $N \phi^{4}$ theory is completely unspectacular and smooth.

Various techniques have been developed to restore the convergence of the perturbative series. The more physically (rather than mathematically) motivated ones include "screened perturbation theory" [8,9], its generalization to gauge theories ("HTL perturbation theory") $[10,11,12,13]$, and the use of $2 \mathrm{PI}$ techniques which put the emphasis on a quasiparticle description [14, 15, 16, 17]. In particular the latter results agree very well with available lattice data down to temperatures about three times the phase transition temperature so that the range of applicability of weak-coupling results does not appear to be restricted to uninterestingly high temperatures. Moreover, these results also agree well in this regime with the perturbative results of Ref. [3, 4] to order $g^{5}$ or even $g^{6} \log (g)$ provided the effective-field-theory parameters used in this approach are not treated strictly perturbatively, but are kept in the form in which they appear naturally [18]. The difference this makes is shown in Fig. 1, where the light-gray band shows the renormalization-scale dependence (a measure of the theoretical uncertainty) to strictly order $g^{5}$, and the medium-gray band shows that of the unexpanded three-loop result, both compared to the lattice result of Ref. [19], represented by the thick dark-gray curve. In the unexpanded three-loop result, the renormalization scale dependence is highly nonlinear such that an extremum is attained at the upper boundary marked PMS for principal of minimal sensitivity ${ }^{1}$; a different optimization of the perturbative result is fastest apparent convergence (FAC), which turns out to be close to the former.

\footnotetext{
${ }^{1}$ A slightly different implementation of the PMS to the thermodynamical potential with roughly comparable results has recently been presented in Ref. [20].
} 


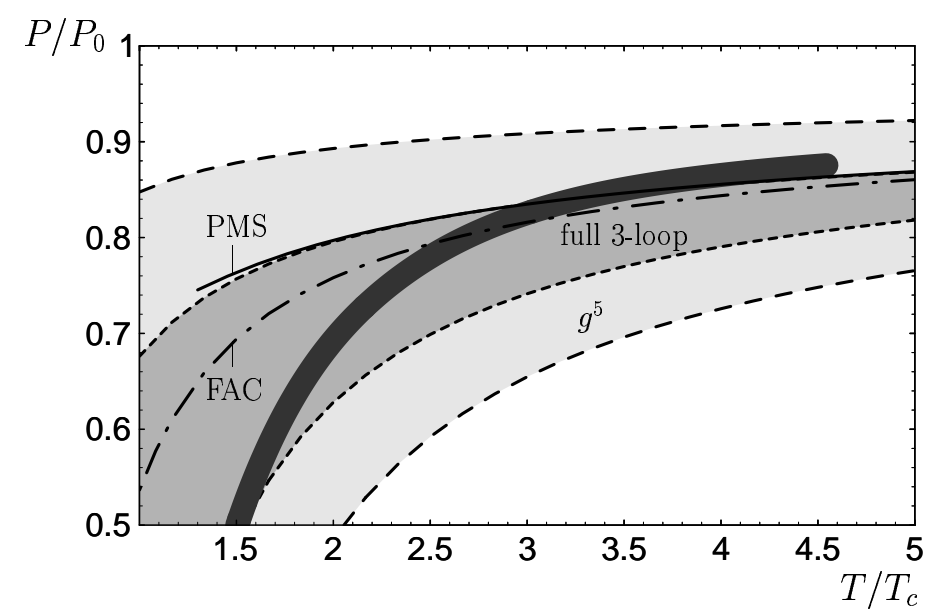

Figure 1: Three-loop pressure in pure-glue QCD normalized to the ideal-gas value with unexpanded effective-field-theory parameters when $\bar{\mu}$ is varied between $\pi T$ and $4 \pi T$ (medium-gray band). The broad light-gray band underneath is the strictly perturbative result to order $g^{5}$ with the same scale variations. The full line gives the result upon extremalization (PMS) with respect to $\bar{\mu}$ (which does not have solutions below $\sim 1.3 T_{c}$ ); the dash-dotted line corresponds to fastest apparent convergence (FAC) in $m_{E}^{2}$, which sets $\bar{\mu} \approx 1.79 \pi T$. (From [18])

In the following, we shall first consider the extension to nonzero quark chemical potential of the perturbative results obtained by Vuorinen by means of the effective field theory provided by the technique of dimensional reduction. After a comparison with lattice results on the one hand, and analytical results from the large- $N_{f}$ limit of QCD on the other hand, we shall turn to the case of chemical potentials much larger than the temperature. This regime turns out to be much richer than the one at high temperature. For sufficiently low temperature, new phases with colour superconductivity occur which can in fact be analysed by weak-coupling techniques applied to full QCD [21, 22, 23, 24, 25, 26, 27]. In this regime, and also in the normal phase at temperatures above the critical one, non-Fermi-liquid effects play a crucial role. As we shall describe, the relevant effective field theory in this situation is those of hard dense loops (HDL) [28, 29], and we have used it to calculate systematically non-Fermi-liquid effects in entropy and specific heat as well as in the dispersion laws of fermionic quasiparticles at low temperature and high quark chemical potential.

\section{Dimensional reduction at high temperature}

When the coupling constant is small, $g \ll 1$, and temperature $T$ is the largest scale, there is a natural hierarchy of scales: the "hard" scale $T$, the "soft" scale $g T$, "ultrasoft" $g^{2} T, \ldots$ In the imaginary-time formalism, the hard scale $T$ is carried by nonzero Matsubara frequencies $\omega_{n}=$ $\pi i n T$ with $n$ even/odd for bosons/fermions, and all the softer scales involve only the zero modes of the bosons. The "soft" scale $g T$ is where collective phenomena such as Debye screening and Landau damping occur ${ }^{2}$ and its effective field theory is Yang-Mills theory reduced to three spatial

\footnotetext{
${ }^{2}$ In plasmas with a momentum-space anisotropy, i.e. temperatures that depend on the direction, the scale $g \max (T)$
} 
dimensions $^{3}$

$$
\mathscr{L}_{E}=\frac{1}{2} \operatorname{tr} F_{i j}^{2}+\operatorname{tr}\left[D_{i}, A_{0}\right]^{2}+m_{E}^{2} \operatorname{tr} A_{0}^{2}+\frac{1}{2} \lambda_{E}\left(\operatorname{tr} A_{0}^{2}\right)^{2}+\ldots
$$

In lowest order one has a dimensionful coupling $g_{E}^{2}=g^{2} T+O\left(g^{4}\right)$ and [38]

$$
m_{E}^{2}=\left(1+N_{f} / 6\right) g^{2} T^{2}+g^{2} \sum_{q} \frac{\mu_{q}^{2}}{2 \pi^{2}}+O\left(g^{4}\right), \quad \lambda_{E}=\frac{9-N_{f}}{12 \pi^{2}} g^{4} T+\ldots
$$

The dominant contributions to the thermodynamic pressure comes from the hard modes, and these contributions are completely perturbative,

$$
P^{\text {hard }}=T^{4}\left(c_{1}+c_{2} g^{2}+c_{3} g^{4}+c_{4} g^{6}+\ldots\right),
$$

though the coefficients $c_{\geq 3}$ depend on the cutoff $\left(\Lambda_{E}\right)$ required to separate hard from soft scales. The soft contributions, on the other hand, are determined by the effective three-dimensional theory, and to three-loop order the result only involves the parameters $g_{E}$ and $m_{E}$ [3]

$$
P_{\text {soft }} / T=\frac{2}{3 \pi} m_{E}^{3}-\frac{3}{8 \pi^{2}}\left(4 \ln \frac{\Lambda_{E}}{2 m_{E}}+3\right) g_{E}^{2} m_{E}^{2}-\frac{9}{8 \pi^{3}}\left(\frac{89}{24}-\frac{11}{6} \ln 2+\frac{1}{6} \pi^{2}\right) g_{E}^{4} m_{E}+\ldots
$$

The complete three-loop pressure of QCD is obtained by adding $P=P^{\text {hard }}+P^{\text {soft }}$, and to achieve the maximal perturbative accuracy $P^{\text {hard }}$ as well as the effective field theory parameters $g_{E}$ and $m_{E}$ are required to order $g^{4}$.

As long as $T \gg m_{E}$, this program can be extended to finite quark chemical potential. Besides modifying the parameters of the effective theory, there are also new, $C$-odd terms in the effective Lagrangian. The one with smallest dimension in nonabelian theories reads $[39,40,41]$

$$
\mathscr{L}_{E}^{(\mu)}=i \frac{g^{3}}{3 \pi^{2}} \sum_{q} \mu_{q} \operatorname{tr} A_{0}^{3}
$$

In general the effects of these additional $C$-odd terms are small compared to the $C$-even operators.

One quantity which is determined to leading order by the operator (2.5) is the flavour offdiagonal quark number susceptibility at zero chemical potential [42]

$$
\chi_{i j} \equiv \frac{\partial^{2} P}{\partial \mu_{i} \partial \mu_{j}}
$$

When quark masses are negligible, all off-diagonal components are equal at $\mu_{i}=0$. Denoting them by $\tilde{\chi}$, the leading-order term involves a logarithmic term coming from the exchange of three electrostatic gluons and is given by [42]

$$
\tilde{\chi} \simeq-\frac{\left(N^{2}-1\right)\left(N^{2}-4\right)}{384 N}\left(\frac{g}{\pi}\right)^{6} T^{2} \ln \frac{1}{g} .
$$

is also the scale of magnetic instabilities [30, 31, 32, 33].

${ }^{3}$ The relevant effective theory for time-dependent problems is still 3+1-dimensional and is given to leading order by the so-called hard-thermal-loop (HTL) effective action [34, 35, 36, 37]. 


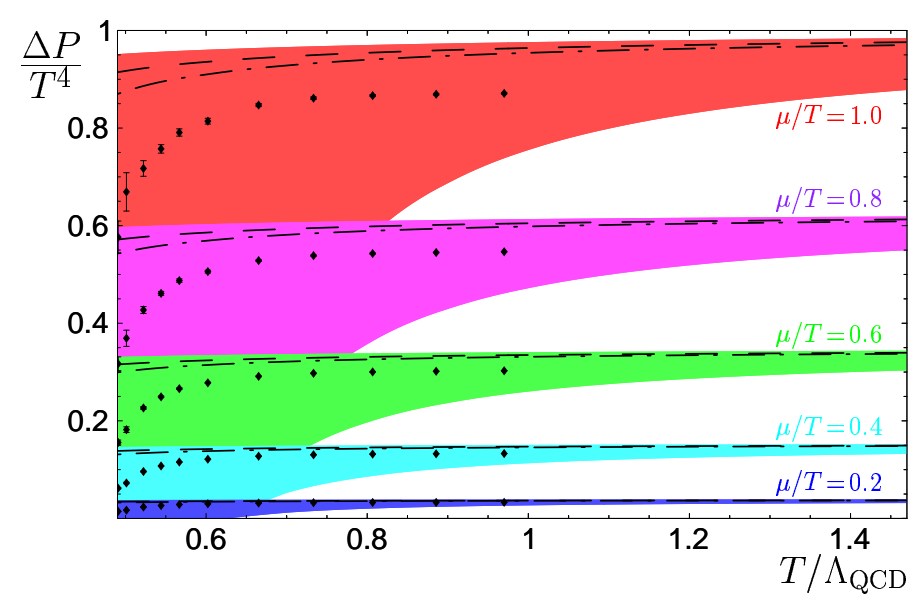

Figure 2: The difference $\Delta P=P(T, \mu)-P(T, 0)$ divided by $T^{4}$ using the unexpanded three-loop result from dimensional reduction of Ref. [48] for $\mu / T=0.2, \ldots, 1.0$ (bottom to top). Shaded areas correspond to a variation of $\bar{\mu}_{\mathrm{MS}}$ around the FAC-m choice by a factor of 2 ; dashed and dash-dotted lines correspond to the FAC-g and FAC-m results, respectively. Also included are the recent lattice data of Ref. [49] (not yet continuum-extrapolated!) assuming $T_{c}^{\mu=0}=0.49 \Lambda_{\mathrm{QCD}}$. (From [54])

where $N$ is the number of colours. This vanishes in SU(2) gauge theory, but not in $\mathrm{QCD}$, and also not in QED, where (in the ultrarelativistic limit) [42]

$$
\left.\tilde{\chi}\right|_{\mathrm{QED}} \simeq-\frac{e^{6}}{24 \pi^{6}} T^{2} \ln \frac{1}{e} \text {. }
$$

There has been for some time a discrepancy of the result (2.7) with lattice results on offdiagonal quark-number susceptibilities. The authors of Refs. $[43,44]$ have obtained results in the deconfined phase that were far below those predicted by perturbation theory, and have interpreted this as new evidence for nonperturbative physics and the failure of weak-coupling methods. Most recent lattice results $[45,46,47]$ have disproved the previous ones, and there is now agreement with the perturbative estimate at $T \geq 2 T_{c}$.

The complete three-loop pressure of QCD at finite quark chemical potential was recently calculated by Vuorinen [48]. Like the result at zero quark chemical potential, there is poor convergence and large renormalization scale dependence at realistic couplings, but again the apparent convergence can be improved importantly by applying the prescription of Ref. [18] which keeps effective-field-theory parameters unexpanded. Figure 2 shows the result for $\Delta P=P(T, \mu)-P(T, 0)$ for $N_{f}=2$ at several values of $\mu / T$ for which there are recent lattice data [49]. The shaded regions correspond to variations of $\bar{\mu}_{\mathrm{MS}}$ by a factor of 2 around a FAC value, and the dashed lines correspond to (two variants of) FAC $\bar{\mu}_{\mathrm{MS}}$. At $T / T_{0}=2$, the highest value considered in [49], the FAC results exceed the not-yet-continuum-extrapolated lattice data consistently by $\approx 10 \%$, which is roughly the expected discretization error [50]. When normalized to the free value $\Delta P_{0}$ instead of $T^{4}$, the results would be essentially $\mu$-independent and thus also very similar to the $N_{f}=2+1$ lattice results of Ref. [51] as well as the quasiparticle model results of Refs. [52, 53].

\section{Large- $N_{f}$ limit of QCD and QED}

While the comparisons of weak-coupling results with lattice data are in remarkably good shape 


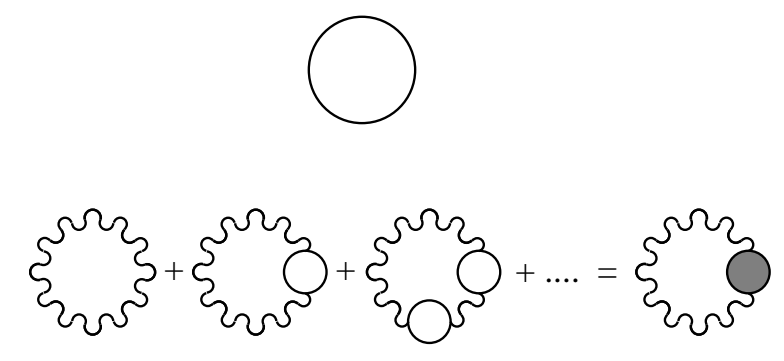

Figure 3: Diagrams contribution to the thermodynamic potential of large- $N_{f}$ QCD

at temperatures a few times the deconfinement temperature, it seems desirable to have a cleaner testing ground for the resummation procedures required in applying weak-coupling techniques at realistic temperatures. Guy Moore [55] has proposed to use the large- $N_{f}$ limit of QCD and QED for this purpose. Large- $N_{f}$ QCD is much simpler than large- $N_{c}$ QCD. In the limit $N_{f} \rightarrow \infty, N_{c} \sim 1$, $g^{2} N_{f} \sim 1$, the relevant diagrams are those displayed in Fig. 3. They involve a dressed gluon propagator which contains typical gauge-theory phenomena such as Debye screening for electrostatic modes, unscreened magnetostatic modes, complicated dispersion laws, Landau damping, and also plasmon damping. This is therefore a much richer theory than the large- $N$ scalar field theories that are frequently used for testing resummations of thermal perturbation theory.

In terms of the polarization tensor $\Pi^{\mu v}=\Pi_{v a c}^{\mu v}+\Pi_{\text {mat }}^{\mu v}$, where the matter part $\Pi_{\text {mat }}^{\mu \nu}$ can be decomposed in a transverse and a spatially longitudinal piece, $\Pi_{T}$ and $\Pi_{L}$, respectively, the thermal pressure reads

$$
\begin{aligned}
P= & N N_{f}\left(\frac{7 \pi^{2} T^{4}}{180}+\frac{\mu^{2} T^{2}}{6}+\frac{\mu^{4}}{12 \pi^{2}}\right) \\
+ & N_{g} \int \frac{d^{3} q}{(2 \pi)^{3}} \int_{0}^{\infty} \frac{d \omega}{\pi}\left[2 \left\{\left[n_{b}+\frac{1}{2}\right] \operatorname{Im} \ln \left(\mathrm{q}^{2}-\omega^{2}+\Pi_{\mathrm{T}}+\Pi_{\mathrm{vac}}\right)\right.\right. \\
& \left.\quad-\frac{1}{2} \operatorname{Im} \ln \left(\mathrm{q}^{2}-\omega^{2}+\Pi_{\mathrm{vac}}\right)\right\} \\
+ & \left.\left\{\left[n_{b}+\frac{1}{2}\right] \operatorname{Im} \ln \frac{\mathrm{q}^{2}-\omega^{2}+\Pi_{\mathrm{L}}+\Pi_{\mathrm{vac}}}{\mathrm{q}^{2}-\omega^{2}}-\frac{1}{2} \operatorname{Im} \ln \frac{\mathrm{q}^{2}-\omega^{2}+\Pi_{\mathrm{vac}}}{\mathrm{q}^{2}-\omega^{2}}\right\}\right]+\mathrm{O}\left(\mathrm{N}_{\mathrm{f}}^{-1}\right)
\end{aligned}
$$

The effective coupling is hidden in $\Pi_{L, T}$ and given by

$$
g_{\mathrm{eff}}^{2}=\left\{\begin{array}{l}
\frac{g^{2} N_{f}}{2}, \mathrm{QCD}, \\
e^{2} N_{f}, \mathrm{QED} .
\end{array}\right.
$$

with exact one-loop beta function:

$$
\frac{1}{g_{\mathrm{eff}}^{2}(\mu)}=\frac{1}{g_{\mathrm{eff}}^{2}\left(\mu^{\prime}\right)}+\frac{\ln \left(\mu^{\prime} / \mu\right)}{6 \pi^{2}} .
$$

There is no asymptotic freedom. In fact, there is a Landau singularity at exponentially large scales $\Lambda_{\mathrm{L}}=\bar{\mu}_{\mathrm{MS}} e^{5 / 6} e^{6 \pi^{2} / g_{\text {eff }}^{2}\left(\bar{\mu}_{\mathrm{MS}}\right)}$, implying this theory exists only as a cutoff theory with $\Lambda_{\text {Cutoff }}<\Lambda_{L}$. 


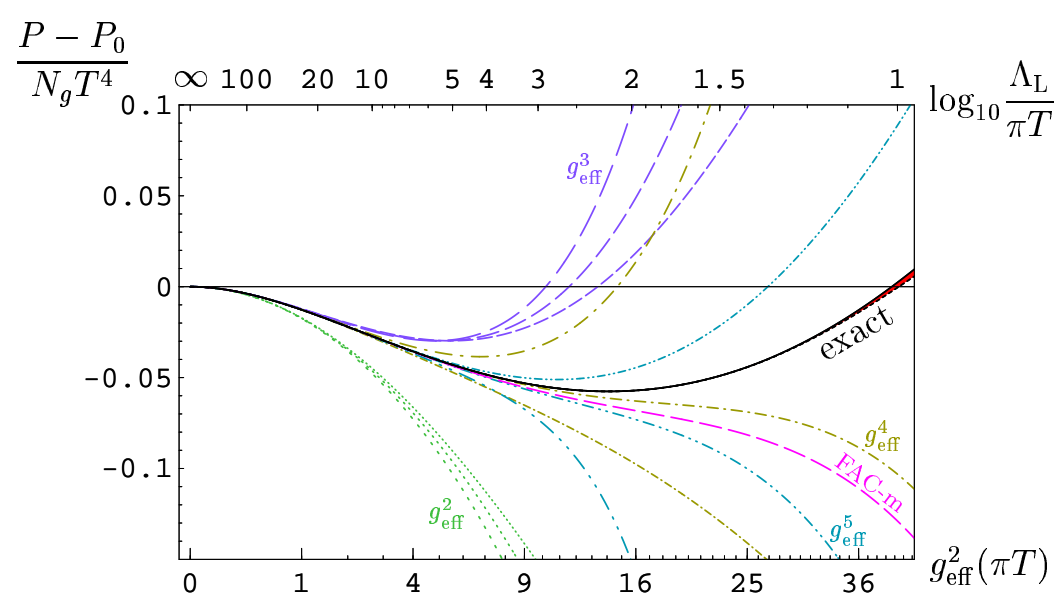

Figure 4: Exact result for the interaction pressure at finite temperature and zero chemical potential at large $N_{f}$ as a function of $g_{\mathrm{eff}}^{2}\left(\bar{\mu}_{\mathrm{MS}}=\pi T\right)$, compared to strict perturbation theory [55, 56, 57]. The tiny red band appearing for large values of the coupling for the exact result shows the cutoff dependence from varying the upper numerical integration cutoff between $50 \%$ and $70 \%$ of the Landau pole $\Lambda_{L}$. The results of strict perturbation theory are given through order $g_{\text {eff }}^{2}$ (dotted line), $g_{\text {eff }}^{3}$ (dashed), $g_{\text {eff }}^{4}$ (dash-dotted), and $g_{\text {eff }}^{5}$ (dashdot-dotted) where the renormalization scale $\bar{\mu}_{\mathrm{MS}}$ is varied between $\frac{1}{2} \pi T$ (line pattern slightly compressed), $\pi T$, and $2 \pi T$ (line pattern slightly stretched). The line labelled "FAC-m" indicates the scale chosen by the prescription of fastest apparent convergence for which the curves of $g_{\text {eff }}^{4}$ and $g_{\text {eff }}^{5}$ coincide. (From [58])

However, for the purpose of testing thermodynamic results this is no problem as long as the effective cutoff provided by temperature and/or chemical potential $T, \mu \ll \Lambda_{\mathrm{L}}$. It does however lead to certain technical intricacies, since $\Lambda_{\text {Cutoff }}$ needs to be implemented such that Euclidean invariances are respected in order not to produce spurious singularities $[55,56]$.

Comparing the available perturbative results with the "exact" large- $N_{f}$ result obtained numerically $[55,56]$ shows that strict perturbation theory has the usual problems of poor convergence and large $\bar{\mu}$-dependences (Fig. 4). The exact result has a curious nonmonotonic behaviour as a function of $g_{\text {eff }}^{2}$, and only the small deviations from the free pressure at small coupling seem to be unambiguously predicted by perturbation theory.

Figure 5 shows the result of an optimized $g^{6}$ result with the prescription of keeping the effective-field-theory parameter $m_{E}$ unexpanded. (The coefficient of the $g^{6}$ term has been extracted numerically with a $10 \%$ error shown by shaded bands.) The result agrees exceedingly well with the exact result even for the largest coupling where the ambiguity introduced by the Landau singularity is still under control.

Perhaps even more remarkably, the nontrivial behaviour of the thermodynamic potential has recently been reproduced with comparable accuracy by using a 2-loop $\Phi$-derivable approximation to the entropy (where the perturbative accuracy is actually only order $g^{3}$ ) [59].

In Ref. [57] two of us have also evaluated the large- $N_{f}$ pressure at finite chemical potential, which is displayed in the 3-d plot of Fig. 6 .

In Fig. 7 this result is compared with the perturbative result to order $g^{5}$ for two optimized renormalization scales. While this shows a fairly large region where the perturbative results obtained 


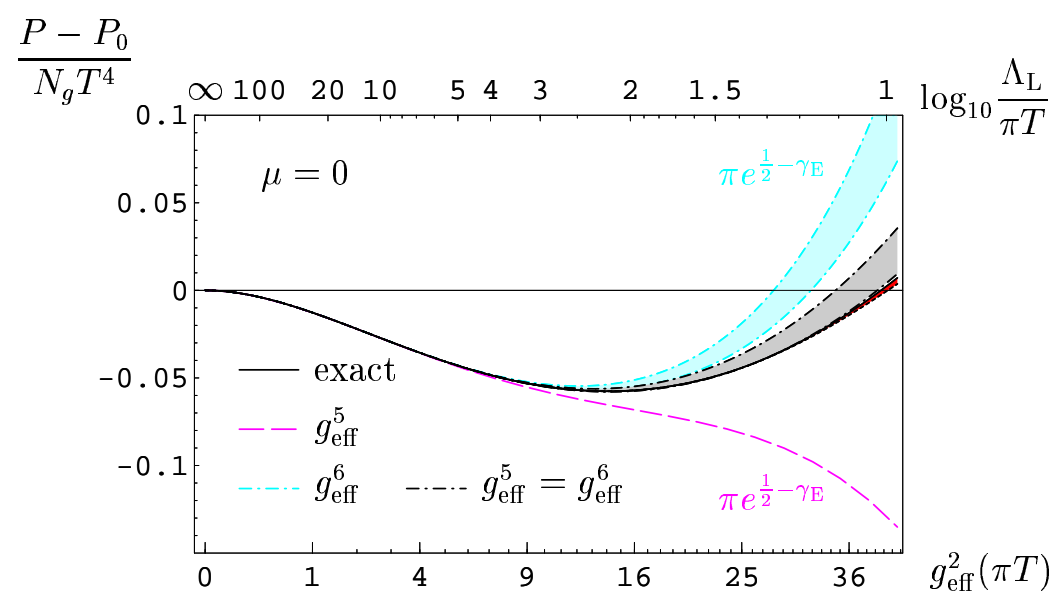

Figure 5: Exact result for the interaction pressure of large- $N_{f}$ QCD at zero chemical potential [55, 56] as a function of $g_{\text {eff }}^{2}\left(\bar{\mu}_{\mathrm{MS}}=\pi T\right)$ or, alternatively, $\log _{10}\left(\Lambda_{\mathrm{L}} / \pi T\right)$. The purple dashed line is the perturbative result when the latter is evaluated with renormalization scale $\bar{\mu}_{\mathrm{MS}}=\bar{\mu}_{\mathrm{FAC}} \equiv \pi e^{1 / 2-\gamma} T$; the blue dash-dotted lines include the numerically determined coefficient to order $g_{\text {eff }}^{6}$ (with its estimated error) at the same renormalization scale. The result marked " $g_{\text {eff }}^{5}=g_{\text {eff }}^{6}$ " corresponds to choosing $\bar{\mu}_{\text {MS }}$ such that the order- $g_{\text {eff }}^{6}$ coefficient vanishes and retaining all higher-order terms contained in the plasmon term $\propto m_{E}^{3}$.

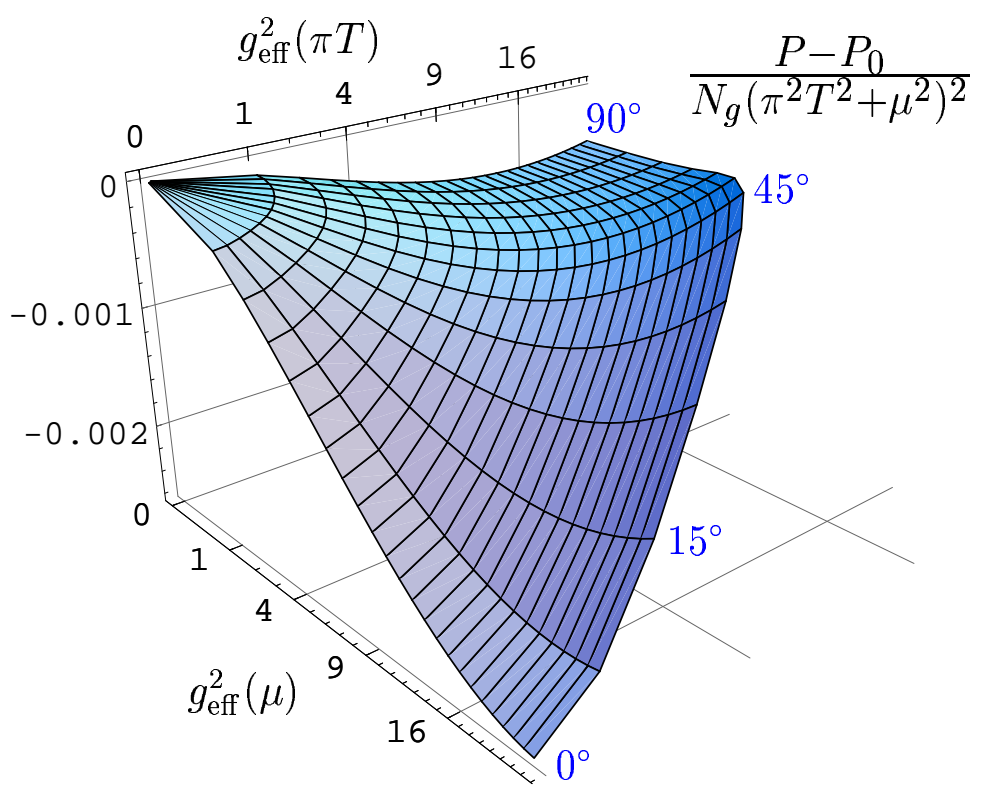

Figure 6: Exact result for the large- $N_{f}$ interaction pressure $P-P_{0}$ normalized to $N_{g}\left(\pi^{2} T^{2}+\mu^{2}\right)^{2}$ as a function of $g_{\text {eff }}^{2}\left(\bar{\mu}_{\mathrm{MS}}\right)$ with $\bar{\mu}_{\mathrm{MS}}^{2}=\pi^{2} T^{2}+\mu^{2}$, which is the radial coordinate, and $\varphi=\arctan (\pi T / \mu)$. 


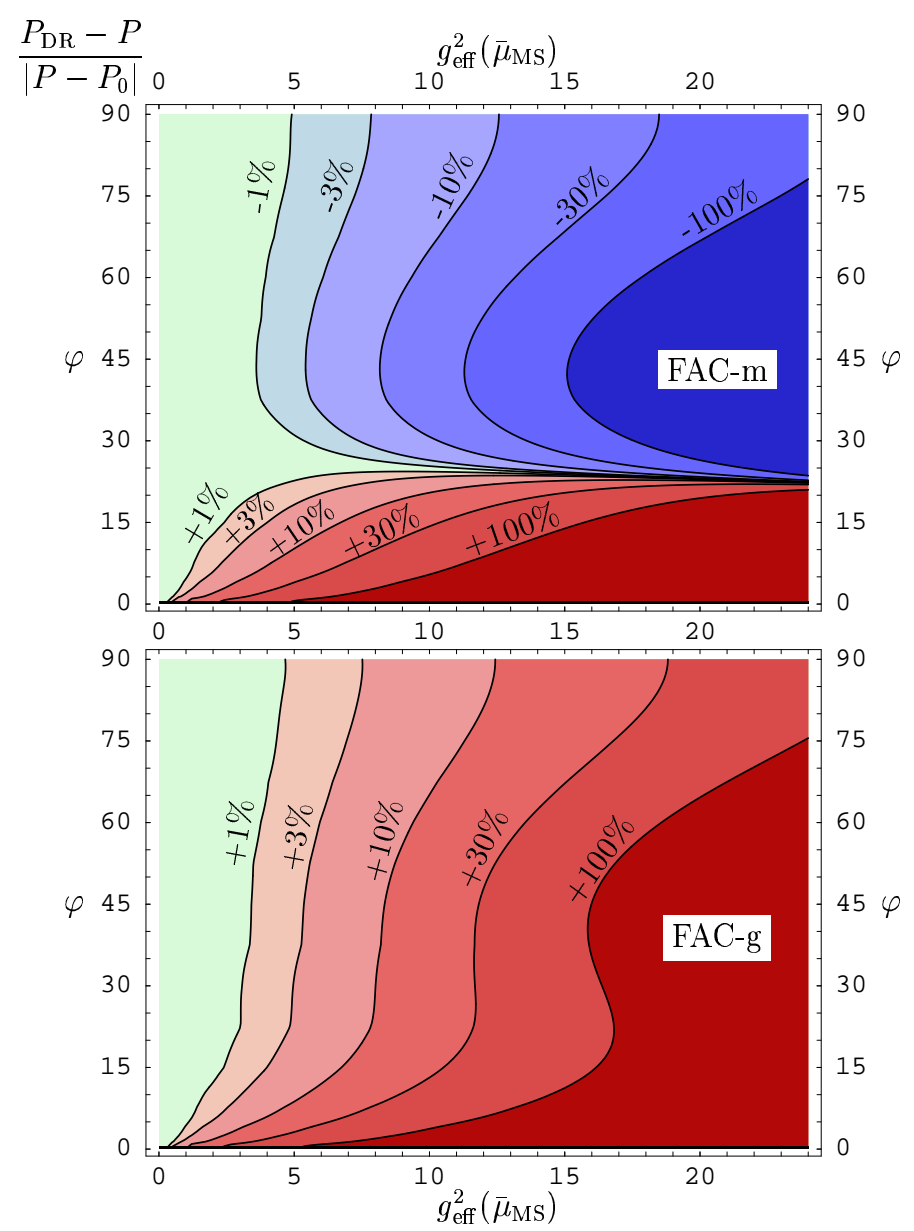

Figure 7: Percentage errors of the perturbative result for the interaction part of the pressure to order $g_{\text {eff }}^{5}$ in the large- $N_{f}$ limit for two choices of $\bar{\mu}_{\mathrm{MS}}$ : Fastest apparent convergence of $P$ as well as $m_{E}^{2}$ (FAC-m), and of $g_{E}^{2}$ (FAC-g). The brightest area corresponds to an error of less than $1 \%$, the darkest ones to an error of over $100 \%$. The ratio of chemical potential to temperature increases from top to bottom according to $\varphi=\arctan (\pi T / \mu)$ so that $90^{\circ}$ corresponds to high temperature and zero chemical potential, and $0^{\circ}$ to zero temperature and high chemical potential. The coupling is given in terms of $g_{\text {eff }}^{2}\left(\bar{\mu}_{\mathrm{MS}}\right)$ at $\bar{\mu}_{\mathrm{MS}}=\sqrt{\pi^{2} T^{2}+\mu^{2}}$. (From [54])

through dimensional reduction is a good approximation, it also shows that there is a breakdown of dimensional reduction at very small temperature $T<0.1 \mu$. In this regime it becomes necessary to evaluate all infrared sensitive diagrams such as ring diagrams in four dimensions. Combining such procedures with the existing analytical results to three-loop order should be able to fill in the existing gap of perturbative results on the pressure for very small temperatures and high chemical potential [60].

A three-loop result for the QCD pressure does exist at exactly zero temperature. It is due to Freedman and McLerran $[61,62,63]$ and it has recently been updated by Vuorinen $[48]^{4}$. In the

\footnotetext{
${ }^{4}$ Another recent update has recently been provided by Ref. [64] where the effects of finite quark masses on the terms of order $g^{2}$ have been computed.
} 


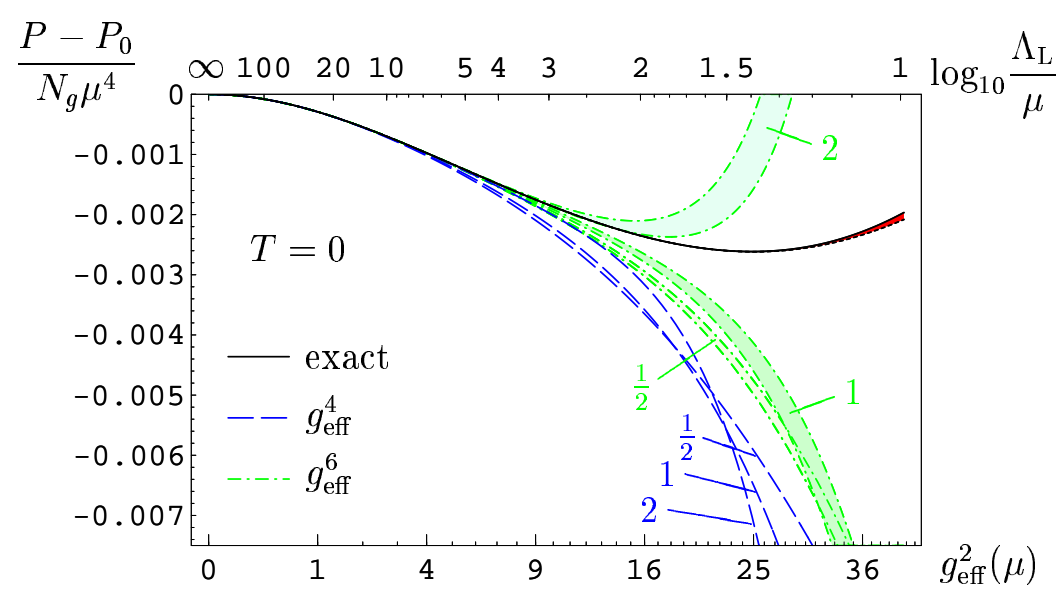

Figure 8: The interaction part of the pressure at zero temperature and finite chemical potential as a function of $g_{\text {eff }}^{2}\left(\bar{\mu}_{\mathrm{MS}}=\mu\right)$ or, alternatively, $\log _{10}\left(\Lambda_{\mathrm{L}} / \mu\right)$, compared with the perturbative result of Freedman and McLerran $[61,63]$ to order $g_{\text {eff }}^{4}$, and our numerically extracted order- $g_{\text {eff }}^{6}$ result, both with renormalization scale in the perturbative results varied around $\bar{\mu}_{\mathrm{MS}}=\mu$ by a factor of 2 . The coloured bands of the $g_{\text {eff }}^{6}$-results cover the error of the numerically extracted perturbative coefficients. (From [57])

large- $N_{f}$ limit it reads

$$
\left.\frac{P-P_{0}}{N_{g} \mu^{4}}\right|_{T=0}=-\frac{g_{\text {eff }}^{2}}{32 \pi^{4}}-\left[\ln \frac{g_{\text {eff }}^{2}}{2 \pi^{2}}-\frac{2}{3} \ln \frac{\bar{\mu}_{\mathrm{MS}}}{\mu}-0.53583 \ldots\right] \frac{g_{\mathrm{eff}}^{4}}{128 \pi^{6}}+O\left(g_{\mathrm{eff}}^{6} \ln g_{\mathrm{eff}}\right) .
$$

The exact (but numerical) large- $N_{f}$ result of Ref. [57] in fact provides a check of these results as well as a numerical determination of the next terms to order $g_{\text {eff }}^{6}$ and $g_{\text {eff }}^{6} \ln \left(g_{\text {eff }}\right)$ :

$$
\left.\frac{P-P_{0}}{N_{g} \mu^{4}}\right|_{g_{\mathrm{eff}}^{6}, T=0, \bar{\mu}_{\mathrm{MS}}=\mu}=\left[3.18(5) \ln \frac{2 \pi^{2}}{g_{\mathrm{eff}}^{2}}+3.4(3)\right] \frac{g_{\mathrm{eff}}^{6}}{2048 \pi^{8}} .
$$

Figure 8 compares the exact and the perturbative results for the large- $N_{f}$ pressure at zero temperature and high chemical potential, with the perturbative results evaluated for three values of $\bar{\mu}_{\mathrm{MS}} / \mu$.

\section{Non-Fermi-liquid behavior}

From the leading-order interaction term in the thermal pressure [65]

$$
P-P_{0}=-N_{g}\left[\frac{5}{9} T^{4}+\frac{2}{\pi^{2}} \mu^{2} T^{2}+\frac{1}{\pi^{4}} \mu^{4}\right] \frac{g_{\text {eff }}^{2}}{32}+\ldots
$$

one would expect that the entropy density $\mathscr{S}=\left(\frac{\partial P}{\partial T}\right)_{\mu}$ at small $T \ll \mu$ should start as

$$
\mathscr{S}-\mathscr{S}_{0}=-N_{g} \frac{g_{\text {eff }}^{2}}{8 \pi^{2}} \mu^{2} T+\ldots
$$

However, for sufficiently small $T$, namely when $T \ll g \mu$, the interaction part of the entropy density extracted from the exact large- $N_{f}$ result (Fig. 9) has even a different sign. The contributions 


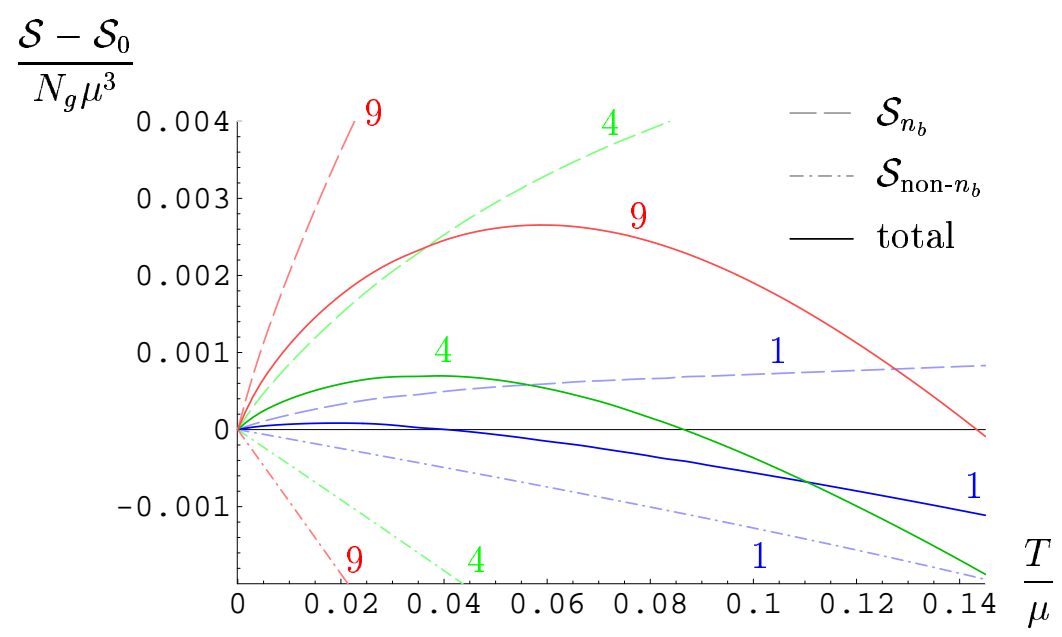

Figure 9: The interaction part of the entropy at small $T / \mu$ for $g_{\text {eff }}^{2}\left(\bar{\mu}_{\mathrm{MS}}=\mu\right)=1,4$, and 9. The "non- $n_{b}$ " contributions (dash-dotted lines) are negative and approximately linear in $T$ with a coefficient agreeing with the exchange term $\propto g_{\text {eff }}^{2}$ in the pressure at small coupling; the " $n_{b}$ " contributions (dashed lines), which are dominated by transverse gauge boson modes, are positive and nonlinear in $T$ such that the total entropy exceeds the free-theory value at sufficiently small $T / \mu$.

to (3.1) which do not involve the Bose distribution function $n_{b}$ do indeed behave in accordance to the result (4.1), but those with the function $n_{b}$ that one would expect to be negligible at very small temperature turn out to become the dominant ones, and they contribute terms which are nonlinear in $T$, implying a strong deviation from the usual behaviour of Fermi liquids [66]. This non-Fermiliquid behaviour is caused by long-range interactions, namely weakly screened magnetic modes. At very small frequencies, the latter have a dynamical screening length $\kappa=\left[\pi m_{\mathrm{D}}^{2} \omega / 4\right]^{1 / 3}$ and it has been found long ago by Holstein, Norton, and Pincus [67] that this manifests itself in the appearance of an anomalous contribution to the low-temperature limit of entropy and specific heat proportional to $\alpha T \ln T^{-1}$.

While this effect is perhaps too small for experimental detection in nonrelativistic situations, it drew renewed theoretical attention more recently $[68,69,70]$ after the detection of non-Fermiliquid behavior in the normal state of high-temperature superconductors [71] and in other systems of strongly correlated electrons, which may be due to effective gauge field dynamics (see also $[72,73,74])$.

In deconfined degenerate quark matter, the analogous effect can more easily be important because the larger coupling constant $\alpha_{s}$ together with the relatively large number of gauge bosons increases the numerical value of the effect by orders of magnitude. In contrast to the case of a hightemperature quark-gluon plasma, chromomagnetostatic fields are expected to remain unscreened in the low-temperature limit [21] and thus lead to the same singularities in the fermion self-energy that are responsible for the breakdown of the Fermi-liquid description in the nonrelativistic electron gas considered in [67].

An important consequence of such non-Fermi-liquid behavior in quantum chromodynamics (QCD) is a reduction of the magnitude of the gap in color superconductors [21, 25, 26, 27] which on the basis of weak-coupling calculations are estimated to have a critical temperature in the range 
between 6 and $60 \mathrm{MeV}$ [75]. Quark matter above this temperature has long-range chromomagnetic interactions that should lead to an anomalous specific heat with possible relevance for the cooling of young neutron stars as pointed out by Boyanovsky and de Vega [76, 77]. However, in Ref. [77] these authors claimed that the $\alpha T \ln T^{-1}$ term in the specific heat as reported in $[67,69,70]$ would not exist, neither in QCD nor in QED. Instead they obtained a $\alpha T^{3} \ln T$ correction to the leading ideal-gas behavior, which by renormalization-group arguments was resummed into a $T^{3+O(\alpha)}$ correction as the leading non-Fermi-liquid effect on the specific heat. ${ }^{5}$ At low temperatures, such a contribution would be rather negligible compared to standard perturbative corrections to the idealgas result $\propto T$.

In Refs. [78, 79] we have recently been able to resolve these contradictory results and shown that the $\alpha T \ln T^{-1}$ term as obtained in $[67,69,70]$ is indeed correct. In addition to the coefficient of this leading logarithm, we have calculated the coefficient under the log as well as higher terms in the low- $T$ series, which come with fractional powers of $T$,

$$
\begin{aligned}
\frac{\mathscr{S}-\mathscr{S}_{0}}{N_{g}}=\frac{g_{\mathrm{eff}}^{2} \mu^{2} T}{36 \pi^{2}}\left(\ln \frac{4 g_{\mathrm{eff}} \mu}{\pi^{2} T}-2+\gamma_{E}-\frac{6}{\pi^{2}} \zeta^{\prime}(2)\right) \\
\quad-\frac{82^{2 / 3} \Gamma\left(\frac{8}{3}\right) \zeta\left(\frac{8}{3}\right)}{9 \sqrt{3} \pi^{11 / 3}}\left(g_{\mathrm{eff}} \mu\right)^{4 / 3} T^{5 / 3}+\frac{802^{1 / 3} \Gamma\left(\frac{10}{3}\right) \zeta\left(\frac{10}{3}\right)}{27 \sqrt{3} \pi^{13 / 3}}\left(g_{\mathrm{eff}} \mu\right)^{2 / 3} T^{7 / 3} \\
+\frac{2048-256 \pi^{2}-36 \pi^{4}+3 \pi^{6}}{540 \pi^{2}} T^{3}\left[\ln \frac{g_{\mathrm{eff}} \mu}{T}-4.3493485 \ldots\right]+O\left(T^{11 / 3}\right) .
\end{aligned}
$$

This expansion is systematic for $T \ll g_{\text {eff }} \mu$, but breaks down at $T \sim g_{\text {eff }} \mu$; for $T \gg g_{\text {eff }} \mu$ it in fact has to switch to the perturbative series that can be obtained from dimensional reduction. A result which covers all $T \ll \mu$ can be obtained by resumming the complete nonlocal HDL self energies and is given by the compact expression [79]

$$
\begin{aligned}
\frac{1}{N_{g}}\left(\mathscr{S}-\mathscr{S}^{0}\right)= & -\frac{g_{\text {eff }}^{2} \mu^{2} T}{24 \pi^{2}}-\frac{1}{2 \pi^{3}} \int_{0}^{\infty} d q_{0} \frac{\partial n_{b}\left(q_{0}\right)}{\partial T} \int_{0}^{\infty} d q q^{2}[ \\
& \left.2 \operatorname{Im} \ln \left(\frac{q^{2}-q_{0}^{2}+\Pi_{T}^{\mathrm{HDL}}}{q^{2}-q_{0}^{2}}\right)+\operatorname{Im} \ln \left(\frac{q^{2}-q_{0}^{2}+\Pi_{L}^{\mathrm{HDL}}}{q^{2}-q_{0}^{2}}\right)\right] \\
& +O\left(g_{\mathrm{eff}}^{4} \mu^{2} T\right) .
\end{aligned}
$$

For $T \ll g \mu$ this resums the above low- $T$ series which is dominated by magnetic effects and for $g_{\text {eff }} \mu \ll T \ll \mu$ connects smoothly to the dimensional reduction result

$$
\frac{1}{N_{g}}\left(\mathscr{S}-\mathscr{S}_{0}\right) \simeq-\frac{g_{\mathrm{eff}}^{2} \mu^{2} T}{8 \pi^{2}}+\frac{g_{\mathrm{eff}}^{3} \mu^{3}}{12 \pi^{4}}
$$

where the $O\left(g^{3}\right)$ contribution is from electrical Debye screening. The numerical evaluation of (4.4) together with the first few orders of the low-temperature series and the perturbative result from dimensional reduction is shown in Fig. 10.

Figure 11 displays the resulting specific heat normalized to its ideal-gas value. The lines marked "QED" correspond to $g_{\text {eff }}=0.303$ or $\alpha_{Q E D} \approx 1 / 137$, and the results for $g_{\text {eff }}=2,3$ correspond to $\alpha_{s} \approx 0.32,0.72$ in two-flavor QCD. (Recall that $g_{\text {eff }}^{2} \equiv g^{2} N_{f} / 2$.) While in QED the effect

\footnotetext{
${ }^{5}$ Resummation of the $\alpha T \ln T^{-1}$ term along the lines of Ref. [77] would have led to a $T^{1+O(\alpha)}$ term instead.
} 


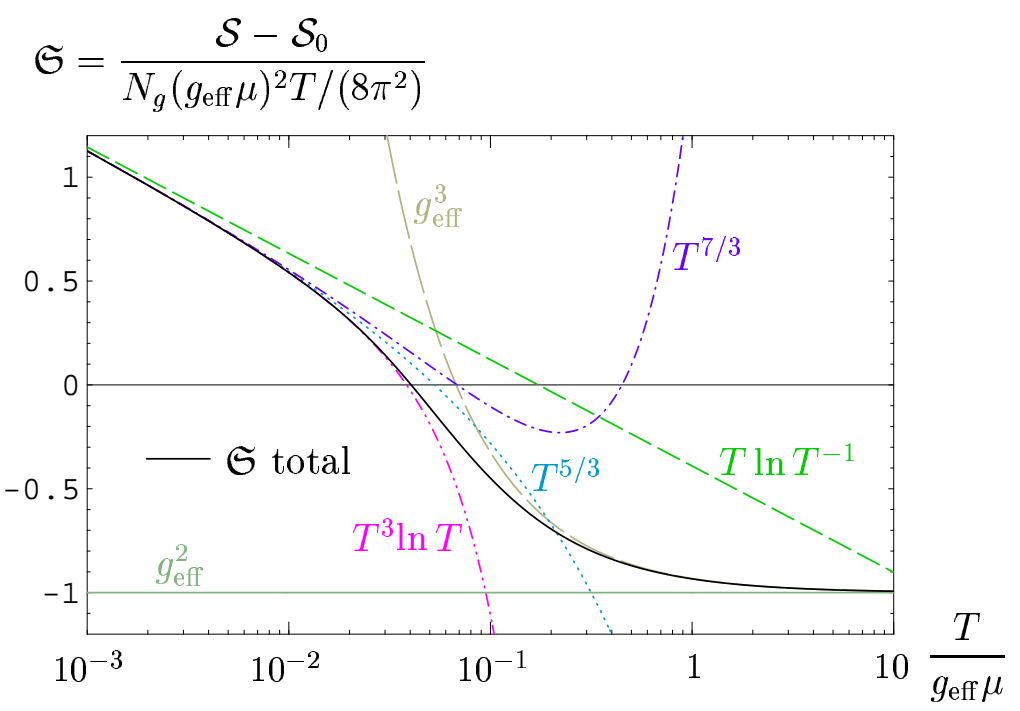

Figure 10: The function $\mathfrak{S}\left(T /\left(g_{\text {eff }} \mu\right)\right)$ which determines the leading-order interaction contribution to the low-temperature entropy. The normalization is such that $\mathfrak{S}=-1$ corresponds to the result of ordinary perturbation theory. The various dash-dotted lines give the first few orders of the low-temperature series for the entropy; the perturbative result from dimensional reduction to order $g_{\text {eff }}^{2}$ and $g_{\text {eff }}^{3}$ is given by the full and the long-dashed curves, resp. (from [79])

is tiny (the deviations from the ideal-gas value have been enlarged by a factor of 20 in Fig. 11 to make them more visible), in QCD we find that there is an interesting range of $T / \mu$ where there is a significant excess of the specific heat over its ideal-gas value, whereas ordinary perturbation theory [65] would have resulted in a low-temperature limit of $\mathscr{C}_{v} / \mathscr{C}_{v}^{0}=1-2 \alpha_{s} / \pi$. At sufficiently low temperature, $\mathscr{C}_{v} / \mathscr{C}_{v}^{0}$ may even become much larger than 1 . As discussed in Ref. [80], the large logarithm that appears in this limit is actually stable against higher-order corrections. However, in QCD (though not in QED) the growth of $\mathscr{C}_{v} / \mathscr{C}_{v}^{0}$ is limited by the appearance of a superconducting phase at sufficiently small $T$. According to Ref. [75], the critical temperature for the color superconducting phase transition may be anywhere between 6 and $60 \mathrm{MeV}$, so with e.g. a quark chemical potential of $\mu=500 \mathrm{MeV}$ the range $T / \mu \geq 0.012$ in Fig. 11 might correspond to normal quark matter with anomalous specific heat. While the effect remains small in QED, it seems conceivable that the anomalous terms in the specific heat play a noticeable role in the thermodynamics of proto-neutron stars, in particular its cooling behavior in its earliest stages before entering color superconductivity $[81,82]$.

The cooling of (proto-)neutron stars with a normal quark matter component is not only sensitive to non-Fermi-liquid effects in the specific heat. The latter are in fact overcompensated by two powers of $\alpha_{s} \ln (m / T)$ appearing in the neutrino emissitivity [83] which come from logarithmic singularities in the group velocities of quark quasiparticles. While Ref. [83] assumed a single scale for the two kinds of non-Fermi-liquid logarithms, a recent explicit calculation [84] showed that these can be very different.

The scale of the logarithm in the specific heat is approximately given by $\log (0.282 \mathrm{~m} / T)$ with $m^{2} \equiv N_{f}(g \mu / 2 \pi)^{2}$. At zero temperature, the group velocity of quark quasiparticles near the (would- 


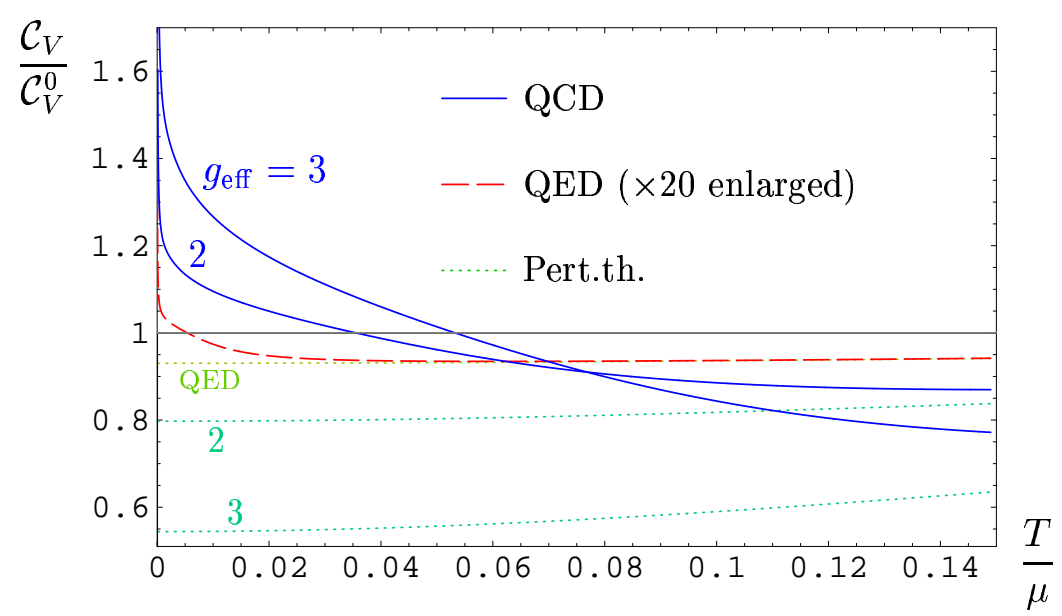

Figure 11: The HDL-resummed result for the specific heat $\mathscr{C}_{v}$, normalized to the ideal-gas value for $g_{\text {eff }}=2$ and 3 corresponding to $\alpha_{s} \approx 0.32$ and 0.72 in two-flavor QCD, and $g_{\text {eff }} \approx 0.303$ for QED. The deviation of the QED result from the ideal-gas value is enlarged by a factor of 20 to make it more visible.

be) Fermi surface $(\varepsilon=E-\mu)$ reads [84]

$$
v_{g}^{-1}(\varepsilon)=1+\frac{g^{2} C_{f}}{12 \pi^{2}} \ln \frac{8.07 m}{|\varepsilon|}+O\left((\varepsilon / m)^{2 / 3}\right)
$$

which at small nonzero temperature $T \ll g \mu$ is bounded by

$$
v_{g}^{-1}(0)=1+\frac{g^{2} C_{f}}{12 \pi^{2}} \ln \frac{9.15 m}{T}+O\left((T / m)^{3}\right) .
$$

Evidently the scale of the latter logarithm differs from that in the anomalous specific heat by more than a factor of 30 .

Higher terms in the small $\varepsilon$ expansion of the group velocity $v_{g}^{-1}(\varepsilon)$ also involve fractional powers, and a complete result for general $\varepsilon$ can again be obtained by HTL resummation. The latter is shown in Fig. 12 together with the first few terms of the small- $\varepsilon$ expansion.

\section{Conclusions}

There has recently been substantial progress in applying weak-coupling methods to the thermodynamics of deconfined QCD, and we have made a case that their generally poor apparent convergence can be greatly improved by suitable resummations. Results obtained from the effective field theory of dimensional reduction remain predictive for temperatures down to a few times the deconfinement temperature (rather than many orders of magnitude higher as previously concluded). This also holds true for nonzero chemical potential $\mu \sim T$, and in fact comparisons with the available lattice data show reasonable agreement already at $T \sim 2 T_{c}$.

For temperatures $T \sim g \mu$ or smaller, the perturbative expansion obtained in the dimensional reduction framework eventually breaks down because of non-Fermi-liquid effects. Those can be calculated analytically by HDL resummation and give rise to anomalous terms in the specific heat and related quantities. While tiny in QED, such effects may become important in the thermodynamics of neutron stars with a normal quark matter component. 


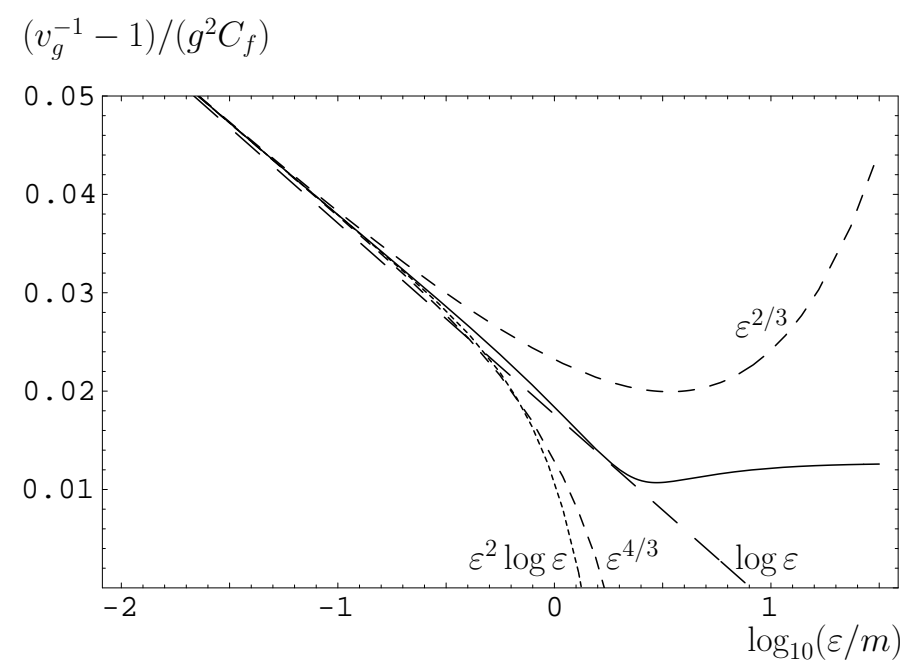

Figure 12: The group velocity of quark quasiparticles plotted as $\left(v_{g}^{-1}-1\right) /\left(g^{2} C_{f}\right)$ over $\log _{10}(E-\mu / m)$ at zero temperature.

\section{Acknowledgments}

This work was supported by the Austrian Science Foundation FWF, project no. P16387-N08.

\section{References}

[1] P. Arnold and C.-X. Zhai, The three-loop free energy for high temperature QED and QCD with fermions, Phys. Rev. D51 (1995) 1906-1918, [hep-ph/9410360].

[2] C.-X. Zhai and B. Kastening, The free energy of hot gauge theories with fermions through $g^{5}$, Phys. Rev. D52 (1995) 7232, [hep-ph/9507380].

[3] E. Braaten and A. Nieto, Free energy of QCD at high temperature, Phys. Rev. D53 (1996) 3421-3437, [hep-ph/9510408].

[4] K. Kajantie, M. Laine, K. Rummukainen, and Y. Schröder, The pressure of hot QCD up to $g^{6} \ln (1 / g)$, Phys. Rev. D67 (2003) 105008, [hep-ph/0211321].

[5] E. Braaten and A. Nieto, Effective field theory approach to high temperature thermodynamics, Phys. Rev. D51 (1995) 6990-7006, [hep-ph / 9501375 ].

[6] R. Parwani and H. Singh, The pressure of hot $g^{2} \phi^{4}$ theory at order $g^{5}$, Phys. Rev. D51 (1995) 4518-4524, [hep-th/9411065].

[7] I. T. Drummond, R. R. Horgan, P. V. Landshoff, and A. Rebhan, Foam diagram summation at finite temperature, Nucl. Phys. B524 (1998) 579-600, [hep-ph/9708426].

[8] F. Karsch, A. Patkós, and P. Petreczky, Screened perturbation theory, Phys. Lett. B401 (1997) 69-73, [hep-ph/9702376].

[9] J. O. Andersen, E. Braaten, and M. Strickland, Screened perturbation theory to three loops, Phys. Rev. D63 (2001) 105008, [hep-ph/ 0007159$].$

[10] J. O. Andersen, E. Braaten, and M. Strickland, Hard-thermal-loop resummation of the free energy of a hot gluon plasma, Phys. Rev. Lett. 83 (1999) 2139-2142, [hep-ph/9902327]. 
[11] J. O. Andersen, E. Braaten, E. Petitgirard, and M. Strickland, HTL perturbation theory to two loops, Phys. Rev. D66 (2002) 085016, [http: / / arXiv.org/abs/hep-ph/0205085].

[12] J. O. Andersen, E. Petitgirard, and M. Strickland, Two-loop HTL thermodynamics with quarks, Phys. Rev. D70 (2004) 045001, [hep-ph/ 0302069$].$

[13] J. O. Andersen and M. Strickland, Resummation in hot field theories, Ann. Phys. 317 (2005) 281-353, [hep-ph/0404164].

[14] J. P. Blaizot, E. Iancu, and A. Rebhan, The entropy of the QCD plasma, Phys. Rev. Lett. 83 (1999) 2906-2909, [hep-ph/9906340].

[15] J. P. Blaizot, E. Iancu, and A. Rebhan, Self-consistent hard-thermal-loop thermodynamics for the quark-gluon plasma, Phys. Lett. B470 (1999) 181-188, [hep-ph/9910309].

[16] J. P. Blaizot, E. Iancu, and A. Rebhan, Approximately self-consistent resummations for the thermodynamics of the quark-gluon plasma: Entropy and density, Phys. Rev. D63 (2001) 065003, [hep-ph/0005003].

[17] J. P. Blaizot, E. Iancu, and A. Rebhan, Thermodynamics of the high-temperature quark-gluon plasma, in Quark-Gluon Plasma 3 (R. C. Hwa and X.-N. Wang, eds.). World Scientific, Singapore, 2003.

[18] J. P. Blaizot, E. Iancu, and A. Rebhan, On the apparent convergence of perturbative QCD at high temperature, Phys. Rev. D68 (2003) 025011, [hep-ph / 0303045 ].

[19] G. Boyd, J. Engels, F. Karsch, E. Laermann, C. Legeland, M. Lütgemeier, and B. Petersson, Thermodynamics of SU(3) lattice gauge theory, Nucl. Phys. B469 (1996) 419-444, [hep-lat/9602007].

[20] M. Inui, A. Niégawa, and H. Ozaki, Improvement of the hot QCD pressure by the minimal sensitivity criterion, Prog. Theor. Phys. 115 (2006) 411-424, [hep-ph / 0501277$].$

[21] D. T. Son, Superconductivity by long-range color magnetic interaction in high-density quark matter, Phys. Rev. D59 (1999) 094019, [hep-ph/9812287].

[22] T. Schäfer and F. Wilczek, Superconductivity from perturbative one-gluon exchange in high density quark matter, Phys. Rev. D60 (1999) 114033, [hep-ph/9906512].

[23] R. D. Pisarski and D. H. Rischke, Gaps and critical temperature for color superconductivity, Phys. Rev. D61 (2000) 051501, [http: / /arXiv.org/abs/nucl-th/9907041].

[24] R. D. Pisarski and D. H. Rischke, Color superconductivity in weak coupling, Phys. Rev. D61 (2000) 074017, [nucl-th/9910056].

[25] W. E. Brown, J. T. Liu, and H.-c. Ren, On the perturbative nature of color superconductivity, Phys. Rev. D61 (2000) 114012, [http: / / arXiv . org/abs/hep-ph/9908248].

[26] W. E. Brown, J. T. Liu, and H.-c. Ren, Non-Fermi liquid behavior, the BRST identity in the dense quark-gluon plasma and color superconductivity, Phys. Rev. D62 (2000) 054013, [http://arXiv.org/abs/hep-ph/0003199].

[27] Q. Wang and D. H. Rischke, How the quark self-energy affects the color-superconducting gap, Phys. Rev. D65 (2002) 054005, [http: / / arXiv . org/abs / nucl-th/0110016].

[28] T. Altherr and U. Kraemmer, Gauge field theory methods for ultradegenerate and ultrarelativistic plasmas, Astropart. Phys. 1 (1992) 133-158.

[29] C. Manuel, Hard dense loops in a cold non-abelian plasma, Phys. Rev. D53 (1996) 5866-5873, [hep-ph/9512365]. 
[30] S. Mrówczyński and M. H. Thoma, Hard-loop approach to anisotropic systems, Phys. Rev. D62 (2000) 036011, [hep-ph/0001164].

[31] P. Romatschke and M. Strickland, Collective modes of an anisotropic quark gluon plasma, Phys. Rev. D68 (2003) 036004, [hep-ph / 0304092$].$

[32] P. Arnold, J. Lenaghan, and G. D. Moore, QCD plasma instabilities and bottom-up thermalization, JHEP 08 (2003) 002, [hep-ph / 0307325$].$

[33] S. Mrówczyński, A. Rebhan, and M. Strickland, Hard-loop effective action for anisotropic plasmas, Phys. Rev. D70 (2004) 025004, [hep-ph/ 0403256 ].

[34] J. Frenkel and J. C. Taylor, High temperature limit of thermal QCD, Nucl. Phys. $\mathbf{B 3 3 4}$ (1990) 199.

[35] E. Braaten and R. D. Pisarski, Soft amplitudes in hot gauge theories: A general analysis, Nucl. Phys. B337 (1990) 569.

[36] J. C. Taylor and S. M. H. Wong, The effective action of hard thermal loops in QCD, Nucl. Phys. B346 (1990) 115-128.

[37] E. Braaten and R. D. Pisarski, Simple effective Lagrangian for hard thermal loops, Phys. Rev. D45 (1992) 1827-1830.

[38] S. Nadkarni, Dimensional reduction in finite temperature quantum chromodynamics. 2, Phys. Rev. D38 (1988) 3287.

[39] C. P. Korthals-Altes, R. D. Pisarski, and A. Sinkovics, The potential for the phase of the Wilson line at nonzero quark density, Phys. Rev. D61 (2000) 056007, [hep-ph/9904305].

[40] A. Hart, M. Laine, and O. Philipsen, Static correlation lengths in QCD at high temperatures and finite densities, Nucl. Phys. B586 (2000) 443-474, [hep-ph / 0004060$].$

[41] D. Bödeker and M. Laine, Finite baryon density effects on gauge field dynamics, JHEP 0109 (2001) 029, [hep-ph/0108034].

[42] J. P. Blaizot, E. Iancu, and A. Rebhan, Quark number susceptibilities from HTL-resummed thermodynamics, Phys. Lett. B523 (2001) 143-150, [hep-ph/ 0110369$].$

[43] R. V. Gavai, S. Gupta, and P. Majumdar, Susceptibilities and screening masses in two flavor QCD, Phys. Rev. D65 (2002) 054506, [http: / / arXiv.org/abs/hep-lat/0110032].

[44] R. V. Gavai and S. Gupta, The continuum limit of quark number susceptibilities, Phys. Rev. $\mathbf{D 6 5}$ (2002) 094515, [http://arXiv.org/abs/hep-lat/0202006].

[45] MILC Collaboration, C. Bernard et. al., Qcd thermodynamics with three flavors of improved staggered quarks, Phys. Rev. D71 (2005) 034504, [hep-lat / 040502 ]].

[46] R. V. Gavai and S. Gupta, The critical end point of QCD, Phys. Rev. D71 (2005) 114014, [hep-lat/0412035].

[47] C. R. Allton et. al., Thermodynamics of two flavor QCD to sixth order in quark chemical potential, Phys. Rev. D71 (2005) 054508, [hep-lat/ 0501030 ].

[48] A. Vuorinen, The pressure of QCD at finite temperatures and chemical potentials, Phys. Rev. D68 (2003) 054017, [hep-ph/0305183].

[49] C. R. Allton, S. Ejiri, S. J. Hands, O. Kaczmarek, F. Karsch, E. Laermann, and C. Schmidt, The equation of state for two flavor QCD at non-zero chemical potential, Phys. Rev. D68 (2003) 014507 , [hep-lat/0305007]. 
[50] F. Karsch, E. Laermann, and A. Peikert, The pressure in 2, 2+1 and 3 flavour QCD, Phys. Lett. B478 (2000) 447, [hep-lat/0002003].

[51] Z. Fodor, S. D. Katz, and K. K. Szabó, The QCD equation of state at nonzero densities: Lattice result, Phys. Lett. B568 (2003) 73-77, [hep-lat/0208078].

[52] K. K. Szabó and A. I. Toth, Quasiparticle description of the QCD plasma, comparison with lattice results at finite T and $\mu, J H E P(\mathbf{0 3 0 6}$ (2003) 008, [hep-ph / 0302255 ].

[53] A. Rebhan and P. Romatschke, HTL quasiparticle models of deconfined QCD at finite chemical potential, Phys. Rev. D68 (2003) 025022, [hep-ph/ 0304294 ].

[54] A. Ipp, A. Rebhan, and A. Vuorinen, Perturbative QCD at non-zero chemical potential: Comparison with the large- $N_{f}$ limit and apparent convergence, Phys. Rev. D69 (2004) 077901, [hep-ph/0311200].

[55] G. D. Moore, Pressure of hot QCD at large $N_{f}$, JHEP 0210 (2002) 055, [hep-ph/ 0209190$]$.

[56] A. Ipp, G. D. Moore, and A. Rebhan, Comment on and erratum to 'Pressure of hot QCD at large $N_{f}$ ', JHEP 0301 (2003) 037, [hep-ph/ 0301057$].$

[57] A. Ipp and A. Rebhan, Thermodynamics of large- $N_{f}$ QCD at finite chemical potential, JHEP 0306 (2003) 032, [hep-ph/0305030].

[58] J.-P. Blaizot, A. Ipp, and A. Rebhan, Study of the gluon propagator in the large- $N_{f}$ limit at finite temperature and chemical potential for weak and strong couplings, hep-ph/0508317.

[59] J.-P. Blaizot, A. Ipp, A. Rebhan, and U. Reinosa, Asymptotic thermal quark masses and the entropy of QCD in the large- $N_{f}$ limit, Phys. Rev. D72 (2005) 125005, [hep-ph / 0509052 ].

[60] A. Ipp, K. Kajantie, A. Rebhan, and A. Vuorinen, The pressure of deconfined QCD for all temperatures and quark chemical potentials, Paper in preparation.

[61] B. A. Freedman and L. D. McLerran, Fermions and gauge vector mesons at finite temperature and density. 2. The ground state energy of a relativistic electron gas, Phys. Rev. D16 (1977) 1147.

[62] B. A. Freedman and L. D. McLerran, Fermions and gauge vector mesons at finite temperature and density. 3. The ground state energy of a relativistic quark gas, Phys. Rev. D16 (1977) 1169.

[63] V. Baluni, Non-abelian gauge theories of Fermi systems: Quantum-chromodynamic theory of highly condensed matter, Phys. Rev. D17 (1978) 2092-2121.

[64] E. S. Fraga and P. Romatschke, The role of quark mass in cold and dense perturbative QCD, Phys. Rev. D71 (2005) 105014, [hep-ph/ 0 412298].

[65] J. I. Kapusta, Finite-temperature field theory. Cambridge University Press, Cambridge, UK, 1989.

[66] L. D. Landau and E. M. Lifshitz, Statistical Physics. Pergamon Press, London, 1958.

[67] T. Holstein, R. E. Norton, and P. Pincus, de Haas-van Alphen effect and the specific heat of an electron gas, Phys. Rev. B8 (1973) 2649-2656.

[68] M. Y. Reizer, Relativistic effects in the electron density of states, specific heat, and the electron spectrum of normal metals, Phys. Rev. B40 (1989) 11571-11575.

[69] J. Gan and E. Wong, Non-Fermi-liquid behavior in quantum critical systems, Phys. Rev. Lett. 71 (1993) 4226-4229.

[70] S. Chakravarty, R. E. Norton, and O. F. Syljuåsen, Transverse gauge interactions and the vanquished Fermi liquid, Phys. Rev. Lett. 74 (1995) 1423-1426. 
[71] C. M. Varma, P. B. Littlewood, S. Schmitt-Rink, E. Abrahams, and A. E. Ruckenstein, Phenomenology of the normal state of Cu-O high-temperature superconductors, Phys. Rev. Lett. 63 (1989) 1996-1999.

[72] J. Polchinski, Effective field theory and the Fermi surface, hep-th/9210046.

[73] J. Polchinski, Low-energy dynamics of the spinon gauge system, Nucl. Phys. B422 (1994) 617-633.

[74] C. Nayak and F. Wilczek, Renormalization group approach to low temperature properties of a nonfermi liquid metal, Nucl. Phys. B430 (1994) 534-562.

[75] D. H. Rischke, The quark-gluon plasma in equilibrium, Prog. Part. Nucl. Phys. 52 (2004) 197-296, [nucl-th/0305030].

[76] D. Boyanovsky and H. J. de Vega, Non-Fermi liquid aspects of cold and dense QED and QCD: Equilibrium and non-equilibrium, Phys. Rev. D63 (2001) 034016, [hep-ph / 0009172 ].

[77] D. Boyanovsky and H. J. de Vega, The specific heat of normal, degenerate quark matter: Non-Fermi liquid corrections, Phys. Rev. D63 (2001) 114028, [hep-ph / 0011354 ].

[78] A. Ipp, A. Gerhold, and A. Rebhan, Anomalous specific heat in high-density QED and QCD, Phys. Rev. D69 (2004) R011901, [hep-ph/ 0309019 ].

[79] A. Gerhold, A. Ipp, and A. Rebhan, Non-Fermi-liquid specific heat of normal degenerate quark matter, Phys. Rev. D70 (2004) 105015, [hep-ph/ 0406087 ].

[80] T. Schäfer and K. Schwenzer, Non-Fermi liquid effects in QCD at high density, Phys. Rev. D70 (2004) 054007, [hep-ph/0 405053$].$

[81] N. Iwamoto, Quark beta decay and the cooling of neutron stars, Phys. Rev. Lett. 44 (1980) 1637-1640.

[82] G. W. Carter and S. Reddy, Neutrino propagation in color superconducting quark matter, Phys. Rev. D62 (2000) 103002, [hep-ph/ 0005228$].$

[83] T. Schäfer and K. Schwenzer, Neutrino emission from ungapped quark matter, Phys. Rev. D70 (2004) 114037, [astro-ph/0410395].

[84] A. Gerhold and A. Rebhan, Fermionic dispersion relations in ultradegenerate relativistic plasmas beyond leading logarithmic order, Phys. Rev. D71 (2005) 085010, [hep-ph/ 0501089 ]. 